\title{
Evaluation of powder-layering vs. spray-coating techniques in the manufacturing of a swellable/ erodible pulsatile delivery system
}

Anastasia Foppoli, Matteo Cerea, Luca Palugan, Lucia Zema, Alice Melocchi, Alessandra Maroni \& Andrea Gazzaniga

To cite this article: Anastasia Foppoli, Matteo Cerea, Luca Palugan, Lucia Zema, Alice Melocchi, Alessandra Maroni \& Andrea Gazzaniga (2020): Evaluation of powder-layering vs. spraycoating techniques in the manufacturing of a swellable/erodible pulsatile delivery system, Drug Development and Industrial Pharmacy, DOI: 10.1080/03639045.2020.1788060

To link to this article: https://doi.org/10.1080/03639045.2020.1788060

Accepted author version posted online: 27 Jun 2020.

Submit your article to this journal $\square$

View related articles

View Crossmark data ¿ᄌ 
Evaluation of powder-layering vs. spray-coating techniques in the manufacturing of a swellable/erodible pulsatile delivery system

Anastasia Foppoli, Matteo Cerea, Luca Palugan, Lucia Zema, Alice

Melocchi, Alessandra Maroni*and Andrea Gazzaniga

Dipartimento di Scienze Farmaceutiche, Università degli Studi di Milano, 20133 Italy

* Corresponding author

Alessandra Maroni

Università degli Studi di Milano

Dipartimento di Scienze Farmaceutiche

Sezione di Tecnologia e Legislazione Farmaceutiche "Maria Edvige Sangalli"

via G. Colombo 71

20133 Milano, Italy

Tel +390250324654

email: alessandra.maroni@unimi.it 


\section{Evaluation of powder-layering vs. spray-coating techniques in the manufacturing of a swellable/erodible pulsatile delivery system}

A swellable/erodible system for oral time-dependent release, demonstrated to provide consistent pulsatile and colonic delivery performance, has been manufactured through a range of coating techniques to achieve the functional hydroxypropyl methylcellulose (HPMC) layer. Although aqueous spray-coating has long been preferred, the processing times and yields still represent open issues, especially in view of the considerable amount of polymer required to give in vivo lag phases of proper duration. In order to make manufacturing of the delivery system more cost-efficient, different coating modes were thus evaluated, namely top and tangential spray-coating as well as powder-layering, using a fluid bed equipment. To this aim, disintegrating tablets of $5 \mathrm{~mm}$ in diameter, containing a tracer drug, were coated up to $50 \%$ weight gain with low-viscosity HPMC, either as a water solution or as a powder formulation. In all eases, process feasibility was assessed following setup of the operating conditions. Irrespective of the technique employed, the resulting dosage forms exhibited uniform coating layers able to defer the onset of release as a function of the amount of polymer applied. The structure and thickness of such layers differed depending on the deposition modes. With respect to top spray-, both tangential spray-coating and powder-layering were shown to remarkably ameliorate the process time, which was reduced to approximately $1 / 3$ and $1 / 6$, and to enhance the yield by almost 20 and 30\%, respectively. Clear advantages associated with such techniques were thus highlighted, particularly with respect to powderlayering here newly proposed for application of a swellable hydrophilic cellulose derivative.

Keywords: oral drug delivery; pulsatile release; colon delivery; spray-coating; tangential-spray rotary fluid bed; top-spray fluid bed; powder-layering; HPMC. 


\section{Introduction}

Pulsatile release is characterized by a time period of no release (lag time) followed by a rapid and complete drug release [1]. It can be classified in multiple-pulse and single-pulse release [2].

An oral system for pulsatile release and time-based colon delivery of drugs was proposed based on a drug-containing core and a functional polymeric coating of relatively high thickness [3]. This layer was obtained from swellable hydrophilic polymers of different viscosity grades, typically hydroxypropyl methylcellulose (HPMC). When exposed to aqueous fluids, the coating undergoes progressive swelling, dissolution and/or erosion, which are responsible for deferring the onset of release as a function of thickness.

Manufacturing of the polymeric layer responsible for the control of release was a challenging step [4]. In the beginning, relatively high-viscosity HPMC grades were selected as coating agents although the experience available on the use of hydrophilic cellulose derivatives for coating purposes was mainly confined to application of thin protective or cosmetic films. Press-coating was initially used to avoid long spraying operations. However, it involved a range of technical problems, chiefly due to difficult centering of the core within the die, thus impacting on coat thickness homogeneity [5]. Moreover, it granted poor versatility in terms of type of core units and coating level. Therefore, spray-coating was attempted [6]. Hydro-ethanolic dispersions of HPMC were first employed to aid nebulization by limiting the viscosity-building effect. The use of an organic solvent, however, posed environmental, safety-related and consequent regulatory problems. In sought of a viable alternative, different grades of HPMC were explored as coating agents in water solution. A 2910 USP low-viscosity HPMC, namely Methocel $^{\circledR}$ E50, yielded acceptable process feasibility and processing time, both in topspray fluid bed and rotating pan, while maintaining the desired ability to delay drug 
release and fine-tune the delay duration, with no major impact on the pulse release rate.

Administered to healthy volunteers, units having Methocel ${ }^{\circledR}$ E50 layer obtained by aqueous spray-coating were shown to provide the desired in vivo performance $[7,8]$. Particularly, reliable colon targeting was achieved when a proper amount of HPMC and an enteric outer film were applied, to cover the whole small intestinal transit time and overcome the variable gastric residence, respectively.

Furthermore, Methocel ${ }^{\circledR}$ E50-coated formulations containing insulin were prepared, and the protein stability was demonstrated not to be impaired by manufacturing $[9,10]$. Adapted to a multiple-unit design, insulin delivery systems were administered to diabetic rats, yielding remarkably increased absorption and reduced glycemic levels as compared to the reference uncoated dosage form [11-13].

Recently, in place of the functional HPMC coating, swellable/erodible standalone capsule shells fabricated by hot-processing techniques, such as injection-molding and fused deposition modelling 3D printing, were also proposed [14-17].

When the functional HPMC layer was obtained by top-spray fluid bed coating, process efficiency was susceptible to improyement, particularly taking account of the relatively thick coats needed to obtain in vivo lag times consistent with pulsatile release and time-dependent colon delivery. Therefore, especially in view of possible industrial scale-up of the system, there was a need for alternative coating techniques that may prove more advantageous with regard to process times and yields. In this respect, tangential-spray rotary fluid bed, so far used for the coating of small-sized units, may constitute an interesting option [18-22]. Typically, pellets are known to be rapidly and efficiently coated, being subject to fast tumbling, ultimately resulting in uniform spiral motion, and hit by the coating solution sprayed tangentially in the same direction.

A further appealing technique to speed up the coating process, capable of 
overcoming most technical issues connected with the HPMC-based coating solutions, may be represented by powder-layering. This is an established process for the manufacturing of pellets that involves the deposition of powdered active pharmaceutical ingredients onto substrate cores [20,23-26]. Such a technique was also recently proposed to fabricate a prolonged-release hydrophilic matrix system through the layering of powder mixtures consisting of drugs and a high-viscosity HPMC onto inert cellulosic seeds $[27,28]$.

With the aim of setting up more convenient manufacturing of the described delivery system, feasibility of potentially advantageous coating techniques, such as the above-mentioned ones, was therefore evaluated in the application of the relevant lowviscosity HPMC layer. Results from the use of a rotary fluid bed apparatus, where coating operations can be performed by either tangential spray-coating (TANsc) or powder-layering (PL), are reported. For comparison purposes, units were also coated by the top-spray fluid bed technique (TOPsc) already employed.

\section{Materials and methods}

\section{Materials}

Acetaminophen direct compression grade (AMP, C.F.M., I) microcrystalline cellulose (Avicel ${ }^{\circledR}$ PH 101, F.M.C., B); sodium starch glycolate $\left(\right.$ Explotab $^{\circledR}$, Penwest

Pharmaceuticals, US); copovidone (Kollidon ${ }^{\circledR}$ VA64, BASF, D); magnesium stearate (Carlo Erba Reagenti, I); colloidal silica (Syloid ${ }^{\circledR} 244$ FP, Grace Davison, US); hydroxypropyl methylcellulose 2910 USP (HPMC, Methocel ${ }^{\circledR}$ E50 Colorcon, UK) polyethylene glycol (PEG 400, Hoechst, UK), talc (Tradeco, I) . 


\section{Manufacturing of tablet cores}

Acetominophen $(80.0 \% \mathrm{w} / \mathrm{w})$, microcrystalline cellulose $(12.5 \% \mathrm{w} / \mathrm{w})$, sodium starch glycolate $(4.5 \% \mathrm{w} / \mathrm{w})$, copovidone $(2.0 \% \mathrm{w} / \mathrm{w})$, colloidal silica $(0.5 \% \mathrm{w} / \mathrm{w})$, and magnesium stearate $(0.5 \% \mathrm{w} / \mathrm{w})$ were mixed in a V-blender (Erweka, D). The mixture was tableted by a rotary press (AM 8S, Officine Ronchi, I) equipped with concave punches (diameter $5 \mathrm{~mm}$, curvature radius $5 \mathrm{~mm}$ ). The tablets were checked for weight (analytical balance BP211D Sartorius Mechatronics, D; $n=20$ ), height and diameter (digital micrometer Mitutoyo, J; n=20), hardness (crushing tester TBH30 Erweka, D; $\mathrm{n}=10$ ), friability (friabilometer TA3R Erweka, D), and disintegration time (threeposition disintegration apparatus DT3 Sotax, $\mathrm{CH}, \mathrm{n}=6$ ). The weight, height, diameter, crushing strength and disintegration time were $79.4 \pm 0.6 \mathrm{mg}, 3.81 \pm 0.02 \mathrm{~mm}, 5.03 \pm 0.01$ $\mathrm{mm}, 74.5 \pm 6.9 \mathrm{~N},<1 \%$ and $<5$ min, respectively.

\section{Coating of tablet cores}

All the processes were carried out by fluid bed equipment (GPCG 1.1, Glatt ${ }^{\circledR} \mathrm{GmbH}, \mathrm{D}$ ) with different configurations according to the coating technique in use. For top-spray coating (TOPsc), a top-spray insert was employed, whereas a rotor insert was utilized for both tangential-spray coating (TANsc) and powder-layering (PL). In the case of PL, a powder-feeding device was also needed.

The coating solution for TOPsc and TANsc was prepared $24 \mathrm{~h}$ prior to use. HPMC (7.5\% w/v) and PEG $400(0.5 \% \mathrm{w} / \mathrm{v})$ were preliminarily dispersed into distilled water at $80^{\circ} \mathrm{C}$ under continuous stirring, and the obtained dispersion was then allowed to cool down at $4{ }^{\circ} \mathrm{C}$ overnight. The coating powder for PL was prepared by mixing HPMC $(93.9 \% \mathrm{w} / \mathrm{w})$, colloidal silica $(1.4 \% \mathrm{w} / \mathrm{w})$ and talc $(4.7 \% \mathrm{w} / \mathrm{w})$ by V-blender. The binding solution contained HPMC 5\% w/v in distilled water and was prepared as described above for the coating solution. 
The process parameters used with all the techniques under investigation were set up through the experimental work and are reported in the Results and Discussion section. Samples of approximately $20 \mathrm{~g}$ were collected during the process every $10 \%$ weight gain step increase. All coated systems were dried overnight in a static oven at 40 ${ }^{\circ} \mathrm{C}$.

\section{Characterization of coated systems}

The coated systems were weighed $(n=20)$, and weight gain (w.g.) was calculated by the following equation:

$$
\text { weight gain } \%=\frac{\overline{\mathrm{m}}_{\text {coated units }}-\overline{\mathrm{m}}_{\text {tablet cores }}}{\overline{\mathrm{m}}_{\text {tablet cores }}} \times 100
$$

where:

$$
\begin{aligned}
& \overline{\mathrm{m}}_{\text {coated units }} \text { is the mean weight of the units after coating } \\
& \overline{\mathrm{m}}_{\text {tablet cores }} \text { is the mean weight of the uncoated tablets }
\end{aligned}
$$

After cross-sectioning, the coated systems were analyzed by digital microscope (Dyno-Lite Pro AM-413T, AnMo Electronics Co., TW) for thickness measurement. Thickness data were the mean of measurements performed in 8 different regions of the HPMC layer of each of 3 coated units. Scanning Electron Microscope (SEM) photomicrographs of cross-sectioned systems were used to randomly confirm the thickness and analyze the structure of the applied coating. Samples were gold-sputtered using a plasma evaporator under vacuum, and the photomicrographs were acquired at an accelerated voltage of $10 \mathrm{kV}$ at 80X magnifications (Leo 1430, Carl Zeiss, CH).

For release studies, an adapted disintegration test method was used. Such a procedure was previously set up and compared to the standard dissolution one in order 
to avoid sticking of the swollen units to the vessels, which was shown to impact on data reliability [29]. A three-position disintegration apparatus was employed, inserting a single dosage unit into each basket-rack assembly so that only one of the 6 available tubes was occupied. The basket-rack assemblies moved in separate vessels at a constant 29 to 32 cycles/min frequency through a $55 \pm 2 \mathrm{~mm}$ distance, immersed in $800 \mathrm{ml}$ of distilled water at $37 \pm 1^{\circ} \mathrm{C}$. Fluid samples were withdrawn automatically at predetermined time points and acetaminophen was quantified by spectrophotometer at 248 nm (Lambda 25, Perkin Elmer, I). Release tests were carried out in triplicate.

\section{RESULTS and DISCUSSION}

\section{Coating process}

A rotary tangential fluid bed equipment was used to identify alternative coating techniques to be used for manufacturing of the previously described system, which may provide shorter processing times and improved yields as compared with conventional top-spraying of aqueous HPMC solutions used so far. By means of this equipment, loading of the polymer onto the substrate cores could indeed be performed either as a solution for spray-coating or as a dry powder for layering.

The rotary apparatus is one of the available types of fluid bed systems where a rotor disk is located at the bottom of the processing chamber (Figure 1a and 1b). The airflow needed for fluidization of cores reaches the chamber through a slot between its internal wall and the disk. The particular combination of the fluidizing air pattern and motion of the disk makes the cores take on a twisting rope-like structure while moving circumferentially as a result of differently acting forces [30,31]. A single spray nozzle is positioned within the side wall of the chamber, so that it may be immersed into the core bed throughout the whole coating process, and the liquid coating formulation may be 
nebulized tangentially to, and in the same direction as, the flow of the cores. For the powder-layering process, a three-way nozzle, able to deliver liquid and powder in close proximity to each other, was employed (Figure 1c).

Disintegrating tablets with appropriate mechanical resistance characteristics were used as substrates for the coating experiments. In order to subsequently evaluate the functionality of the applied coatings, AMP was incorporated into the cores as an analytical tracer drug. The units were coated up to a final weight gain of $50 \%$ in batches having the same nominal size of $1 \mathrm{~kg}$. In all cases, the processing conditions needed to be set up in order to achieve effective motion of the cores, deposition of the coating material and solvent removal (Table I).

In the case of spray-coating, an aqueous $7.5 \% \mathrm{w} / \mathrm{v}$ solution of Methocel ${ }^{\circledR}$ E50, plasticized with PEG, was used as the coating formulation, as established through previous research work [32]. Although attempts at increasing the HPMC concentration were made, less diluted solutions brought about the need for reducing the spray rate and caused problems of clogging of the nozzle, thus involving frequent interruptions for cleaning operations.

The coating solution was maintained at $55{ }^{\circ} \mathrm{C}$ since this enabled, by decreasing viscosity, an acceptable spray rate to be set and maintained without major technical problems throughout the whole coating process.

For both TOPsc and TANsc, setup of operating conditions was performed firstly taking into account the need for ensuring adequate motion of the cores inside the equipment. Therefore, it was necessary to adjust the inlet air flow and, in the case of TANsc, also the disk rotation speed so as to avoid mechanical damages to the units, especially at the beginning of the process. The spray rate was adjusted in sought of a proper balance between wetting and drying, which differed according to whether top- or 
tangential-spray mode was used. Coupled with adequate selection of the inlet air temperature, this allowed feasible processing to be performed by preventing sticking, clogging and powdering problems. Indeed, under the selected conditions, coating by both top- and tangential-spray modes was carried out straightforwardly with limited disruptions for nozzle cleaning or removal of core aggregates. Only in the case of TOPsc, the process was occasionally interrupted in order to restore free liquid flowing through the nozzle and full functionality of the sleeve filters for outlet air.

The amount of HPMC coating solution employed was relatively high when referred to the batch size, regardless of whether it was sprayed from top or tangentially to the fluidized core bed.

In the case of PL, formulation of the powder mixture to be stratified represented in principle the most challenging step. The coating powders had to show adequate flow properties for consistent dosing into the process chamber and for uniform distribution onto the substrates. These were indeed prerequisites for attainment of homogeneous coating layers and acceptable process yields. Flowability testing of the Methocel ${ }^{\circledR}$ E50 batch in use, however, demonstrated that the particle size was not so critical in this particular case. Flow properties could also be improved by the addition of colloidal silica at $1.5 \% \mathrm{w} / \mathrm{w}$, as confirmed by Carr index values. The $>125 \mu \mathrm{m}$ sieved fraction, which covered no more than $10 \%$ by weight of the HPMC powder, was discarded only, to prevent any impact on structural homogeneity of the applied layer. Preliminary coating trials indicated the need for including an anti-tacking agent into the powder formulation to reduce the observed tendency to the formation of core aggregates and sticking of the units to the internal wall of the equipment. Talc at $5 \% \mathrm{w} / \mathrm{w}$ on the polymer mass was demonstrated to overcome such issues also enhancing flowability of the powder mixture. 
The aqueous binding solution, sprayed to have the polymer particles stuck on the substrate, was obtained by employing the same HPMC grade used as the coating agent at a concentration of $5 \% \mathrm{w} / \mathrm{v}$, which helped growth of the coating layer without hindering nebulization. Prior to powder addition, a sealing phase was carried out, during which only the binding solution was sprayed. After mild drying, a thin film was formed that would serve as a protective barrier against possible mechanical damages and moisture penetration into the drug-containing units during subsequent layering operations. The resulting sealing film also promoted initial adhesion of the particles to the core surface when powder dosing was started. Afterwards, addition of the solid material and nebulization of the binding solution were simultaneously run, making the layer grow consistently during the process. In this phase, the inlet air temperature was diminished to $30{ }^{\circ} \mathrm{C}$ in order to limit solvent drying, thus allowing sufficient adhesiveness to be maintained. A product temperature of $24-26^{\circ} \mathrm{C}$ was consistently recorded in-process, thus indicating that any phenomena of heat transfer to the substrate possibly brought about by impaction may have been offset by continuous cooling due to endothermic evaporation of water from the binding solution. When the desired coating level was achieved, corresponding to $50 \%$ w.g., a drying step was necessary to enable removal of excess humidity from the product at the end.

As expected, PL involved the use of far lower volumes of liquids as compared with each of the spray-coating techniques. Particularly, the total amount of water was less than $1 / 5$ as compared with that required by TANsc and TOPsc. Entailing relatively small amounts of aqueous HPMC solution to have a coating structure formed from the polymer powder, PL was far less burdened than spray-coating by the time- and energyconsuming solvent evaporation step. Accordingly, the time needed to reach $50 \% \mathrm{w} . \mathrm{g}$. 
was considerably reduced, the coating process taking 100 min only vs. 270 min and 791 min necessary with TANsc and TOPsc, respectively.

The relationship between processing time taken by all the techniques under investigation and coating level reached, expressed as weight gain of the units, was found linear $\left(\mathrm{R}^{2}>0.99\right)$, as highlighted in Figure 2. Such durations merely refer to spraying and layering phases. Sporadic interruptions for cleaning of the nozzle and filters, or for programmed visual inspection and sample withdrawal, were not accounted for, neither were the initial and final conditioning operations, i.e. preheating and cooling. In the case of PL, however, a time lapse of 30 min was also involved for final drying, thus making the process last 130 min overall.

Notably, TANsc and PL process times could both be reduced as compared with TOPsc, being about $34 \%$ and $16 \%$ of the latter, respectively. Due to the interplay between the peculiar position of the nozzle, directly immersed within the fluidized core bed, and the fluidizing air pattern, TANsc allowed the coating solution to be sprayed at a higher rate than TOPsc. Indeed, not only the solvent may have been removed from the coated units more effectively, but also the short distance separating the cores from the nozzle orifice would have promoted fast and efficient deposition of the coating droplets, thus lessening premature solvent evaporation. Powdering phenomena inside the process chamber, leading to a decreased amount of polymer properly loaded onto the substrate, may have thereby been limited. Moreover, equicurrent motion of the cores and of the nebulized coating liquid may have favored prompt dragging of the latter from the nozzle port, thus preventing possible clogging and formation of filaments as well as fringes of dried HPMC in its close proximity.

As regards the process yield, expressed as the percent ratio between the amount of coating material actually loaded onto the core units and that used throughout the 
process, TANsc was proved superior to TOPsc. Indeed, the already discussed advantages associated with the position of the nozzle in the rotary fluid bed equipment would enable lower waste of coating material in the form of clogs and filaments at the nozzle port, free powder within the processing chamber and films partly lining its inner wall. With PL, although spreading of the coating powder dragged by the airflow and unproper aggregation of particles had to be accounted for, losses connected with nozzle clogging and polymer sheet formation were fully overcome, thus further improving the overall yield.

\section{Characterization of the coated tablets}

Units with increasing coating levels, collected during TOPsc, TANsc and PL, were characterized, showing reproducible weight and coating thickness (Table II). A smooth aspect was exhibited by spray-coated samples. In contrast, the surface of coatings applied by PL was rather rough, and a somewhat more rounded shape was taken on as weight was gained by the units, partly masking the cylindrical geometry of the original cores when $50 \%$ w.g. was reached. The layers obtained by all the techniques under investigation, as highlighted by SEM analysis of cross-sectioned coated units, turned out continuous and uniform in aspect as well as thickness (Figure 3). Remarkable differences in the relevant structure, reasonably associated with the diverse physical forms and deposition modes of the coating material, are evident in the photomicrographs reported.

Coatings resulting from the same nominal mass of polymer applied by TOPsc, TANsc and PL showed different thickness, which was higher in the order PL>TOPsc > TANsc at all coating levels included in the considered 10-50\% w.g. range (Figure 4). This would be connected with a different extent of layer densification 
depending on the technique employed. Indeed, PL necessarily involved inherent porosity of the coating that consisted of powder particles stuck together. On the other hand, spraying of coating solutions, either top o tangential, led to less porous layers because the relevant growth was due to progressive formation of a packed polymer matrix while the solvent was removed by evaporation. The greater apparent density exhibited by TANsc- vs. TOPsc-derived coatings may have resulted from more frequent, higher impact energy in-process collisions undergone by the units owing to centrifugal acceleration associated with rotation of the disk. Cumulative effect of such mechanical stresses made TANsc layers progressively thin over TOPsc and PL ones as weight gain rose. With each technique, the relationship between coating thickness and weight gain of the coated tablets was found linear, at least in the range of coating levels investigated.

Release testing of the coated units was performed in order to assess the functionality of the coatings applied and compare their efficiency. Irrespective of which of the three techniques was used, the HPMC layer was proved capable of deferring release of the drug from the tablet core. The duration of delay was dependent on the coating level. At the end of the lag time, release was fast and quantitative, coinciding with the observed disintegration of the units. This behaviour was analogous at any of the investigated weight gains. By way of example, the release profiles from systems coated up to $30 \%$ and $50 \%$ w.g. are shown in Figure 5.

A linear relationship was found between lag time, expressed as $\mathrm{t}_{10 \%}$, and the weight gain of the units following coating by TOPsc, TANsc and PL (Figure 6). The release performance of PL-coated tablets having the lowest weight gain investigated, i.e. $10 \%$, could not be distinguished from that of the uncoated ones. However, as the coating level increased, the efficiency of the HPMC barrier manufactured by PL was 
improved, turning out even higher than observed with TOPsc and TANsc at 50\% w.g. These findings may be due to the more porous structure of PL coatings, which would differently affect the relevant performance depending on the amount of polymer applied. Indeed, when the latter is relatively low, it may not be sufficient to establish a continuous protective barrier upon polymer swelling. On the other hand, greater thickness of PL vs. TOPsc and TANsc coatings would be reflected in relative extension of the path to be covered by inward water diffusion, which would counteract the discontinuous nature of the polymer layer. The inherently less effective performance of the PL coating below $40 \%$ w.g. might thus have been outweighed by lengthening of the diffusional path at higher values, as here observed with $50 \%$ w.g. The layers obtained by the two spray-coating techniques provided comparable increase in $\mathrm{t}_{10 \%}$ as a function of weight gain, although delay times yielded by the TOPsc one were longer. Also in this case, as argued for PL, higher thickness of the TOPsc coating per amount of polymer applied, due to a greater extent of porosity, could justify its better efficiency $v s$. TANsc.

In order to compare the different coating techniques taking account of both process time and ability to defer drug release of the resulting polymer layers, a dimensionless parameter previously introduced was used, i.e. the Time Equivalent Process Parameter (TEPP) [32]. Indeed, TEPP is given by the ratio between process time and in vitro lag time, expressed as $\mathrm{t}_{10 \%}$, thus indicating the processing time required to achieve one lag time unit. Accordingly, decreasing TEPP values would point out increasing time efficiency of the process. With TOPsc, TANsc, and PL, TEPP of 13.8, 5.7 and 1.6 was obtained, respectively. In light of these results, both PL and TANsc were shown remarkably more convenient processing modes over TOPsc for application of the functional HPMC coating. Particularly, an almost ten-fold time efficiency was achieved when operating by PL, which was also coupled with the highest process yield. 


\section{CONCLUSIONS}

For the purpose of attaining the swellable/erodible polymeric layer of a previously-described system intended for time-dependent release, different coating modes have comparatively been evaluated using a fluid bed equipment. Particularly, aqueous spray-coating, either under top- or tangential-spray configuration of the apparatus, and powder-layering were explored. All processes turned out feasible and provided coated units having satisfactory physico-technological characteristics as well as the desired in vitro release profiles. Powder-layering was proved largely advantageous over the other techniques under investigation, in view of the relatively small volume of liquid to be removed and the limited polymer losses. Indeed, it allowed a more than six-fold and two-fold reduction in the processing time as compared with top and tangential spray-coating, respectively. To a lesser extent, the yield was also improved.

Despite the use of the same polymer solution, coating efficiency was greater with the tangential- rather than the top-spraying mode, processing time and yield both being ameliorated. Notably, the former was reduced to approximately $1 / 3$ and the latter was increased by nearly $20 \%$.

Based on the above findings, tangential spray-coating and powder-layering turned out to be valuable alternatives with respect to top spray-coating mostly used so far in the manufacturing of the delivery system described. Powder-layering results were particularly interesting given the fully novel application of such a technique, never employed before for application of coating layers based on hydrophilic cellulose derivatives. This could not only allow the coating step to be considerably improved but also enable exploitation of other hydrophilic polymers with higher viscosity 
characteristics, previously unexplored or discarded because of technical difficulties encountered in the nebulization of the relevant aqueous solutions.

\section{REFERENCES}

[1] Bussemer T, Otto I, Bodmeier R. Pulsatile Drug-Delivery Systems. Crit. Rev. Ther. Drug Carr. Syst. 2001;18:433-458.

[2] Bussemer T, Peppas NA, Bodmeier R. Evaluation of the swelling, hydration and rupturing properties of the swelling layer of a rupturable pulsatile drug delivery system. Eur. J. Pharm. Biopharm. 2003;56:261-270.

[3] Gazzaniga A, Giordano F, Sangalli ME, et al. Oral colon-specific drug delivery: Design strategies. S.T.P. Pharma Prat. 1994;4:336-343.

[4] Maroni A, Zema L, Loreti G, et al. Film coatings for oral pulsatile release. Int. J. Pharm. 2013;457:362-371.

[5] Foppoli AA, Maroni A, Cerea M, et al. Dry coating of solid dosage forms: an overview of processes and applications. Drug Dev. Ind. Pharm. 2017;43:1919-1931.

[6] Maroni A, Zema L, Cerea M, et al. Erodible drug delivery systems for timecontrolled release into the gastrointestinal tract. J. Drug Deliv. Sci. Technol. 2016;32:229-235.

[7] Sangalli ME, Maroni A, Zema L, et al. In vitro and in vivo evaluation of an oral system for time and/or site-specific drug delivery. J. Control. Release. 2001;73:103110.

[8] Foppoli A, Maroni A, Moutaharrik S, et al. In vitro and human pharmacoscintigraphic evaluation of an oral 5-ASA delivery system for colonic release. Int. J. Pharm. 2019;572:118723.

[9] Maroni A, Del Curto MD, Serratoni M, et al. Feasibility, stability and release performance of a time-dependent insulin delivery system intended for oral colon release. Eur. J. Pharm. Biopharm. 2009;72:246-251. 
[10] Del Curto MD, Maroni A, Palugan L, et al. Oral delivery system for two-pulse colonic release of protein drugs and protease inhibitor/absorption enhancer compounds. J. Pharm. Sci. 2011;100:3251-3259.

[11] Maroni A, Del Curto MDMD, Cerea M, et al. Polymeric coatings for a multipleunit pulsatile delivery system: Preliminary study on free and applied films. Int. J. Pharm. 2013;440:256-263.

[12] Del Curto MD, Palugan L, Foppoli A, et al. Erodible time-dependent colon delivery systems with improved efficiency in delaying the onset of drug release. J. Pharm. Sci. 2014;103:3585-3593.

[13] Maroni A, Del Curto MD, Salmaso S, et al. In vitro and in vivo evaluation of an oral multiple-unit formulation for colonic delivery of insulin. Eur. J. Pharm. Biopharm. 2016;108:76-82.

[14] Zema L, Loreti G, Macchi E, et al. Injection-molded capsular device for oral pulsatile release: Development of a novel mold. J. Pharm. Sci. 2013;102:489-499.

[15] Macchi E, Zema L, Maroni A, et al. Enteric-coating of pulsatile-release HPC capsules prepared by injection molding. Eur. J. Pharm. Sci. 2015;70:1-11.

[16] Maroni A, Melocchi A, Parietti F, et al. 3D printed multi-compartment capsular devices for two-pulse oral drug delivery. J. Control. Release. 2017;268:10-18.

[17] Melocchi A, Parietti F, Maccagnan S, et al. Industrial Development of a 3D-Printed Nutraceutical Delivery Platform in the Form of a Multicompartment HPC Capsule. AAPS PharmSciTech. 2018;19:3343-3354.

[18] Jones D. Air Suspension Coating for Multiparticulates. Drug Dev. Ind. Pharm. 1994;20:3175-3206.

[19] Vecchio C, Fabiani F, Sangalli ME, et al. Rotary tangential spray technique for aqueous film coating of indobufen pellets. Drug Dev. Ind. Pharm. 1998;24:269-274.

[20] Vuppala MK, Parikh DM, Bhagat HR. Application of powder-layering technology and film coating for manufacture of sustained-release pellets using a rotary fluid bed processor. Drug Dev. Ind. Pharm. 1997;23:687-694. 
[21] Yang ST, Van Savage G, Weiss J, et al. The effect of spray mode and chamber geometry of fluid-bed coating equipment and other parameters on an aqueous-based ethylcellulose coating. Int. J. Pharm. 1992;86:247-257.

[22] Jones DM, Rajabi-Siahboomi AR. Fluid Bed Technology, Process Robustness, and Scale-Up. In: Rajabi-Siahboomi AR, editor. Multiparticulate Drug Deliv. Formul. Process. Manuf. New York, NY: Springer New York; 2017. p. 65-93.

[23] Nastruzzi C, Cortesi R, Esposito E, et al. Influence of formulation and process parameters on pellet production by powder layering technique. AAPS PharmSciTech. $2000 ; 1$.

[24] Kovacevic J, Mladenovic A, Djuris J, et al. Evaluation of powder, solution and suspension layering for the preparation of enteric coated pellets. Eur. J. Pharm. Sci. 2016;85:84-93.

[25] Li F, Zheng X, Bao Y, et al. Fenofibrate modified-release pellets with lag phase and high oral bioavailability. Drug Des. Devel. Ther. 2019;13:141-151.

[26] Tomuta I, Leucuta SE. Use of experimental design for identifying the most important formulation and technological variables in pelletization by powder layering. J. Drug Deliv. Sci. Technol. 2004;14:215-221.

[27] Cerea M, Maroni A, Palugan L, et al. Novel hydrophilic matrix system with nonuniform drug distribution for zero-order release kinetics. J. Control. Release. 2018;287:247-256.

[28] Cerea M, Foppoli A, Palugan L, et al. Non-Uniform Drug Distribution Matrix System (NUDDMat) for Zero-Order Release of Drugs with Different Solubility. Int. J. Pharm. 2020;119217.

[29] Zema L, Maroni A, Foppoli A, et al. Different HPMC viscosity grades as coating agents for an oral time and/or site-controlled delivery system: An investigation into the mechanisms governing drug release. J. Pharm. Sci. 2007;96:1527-1536.

[30] Dixit R, Puthli S. Fluidization Technologies: Aerodynamic Principles and Process Engineering. J. Pharm. Sci. 2009;98:3933-3960. 
[31] Hadfield J P. Processing and Equipment Considerations for Aqueous Coatings. In: Linda A. Felton, editor. Aqueous Polym. Coatings Pharm. Dos. Forms. 4th ed. Boca Raton: CRC Press; 2016. p. 34.

[32] Sangalli ME, Maroni A, Foppoli A, et al. Different HPMC viscosity grades as coating agents for an oral time and/or site-controlled delivery system: A study on process parameters and in vitro performances. Eur. J. Pharm. Sci. 2004;22:469-473.

\section{Acknowledgment}

SEM analysis was carried out at NOLIMITS, an advanced imaging facility established by Università degli Studi di Milano.

\section{Disclosure of interest}

The authors report no conflict of interest. 

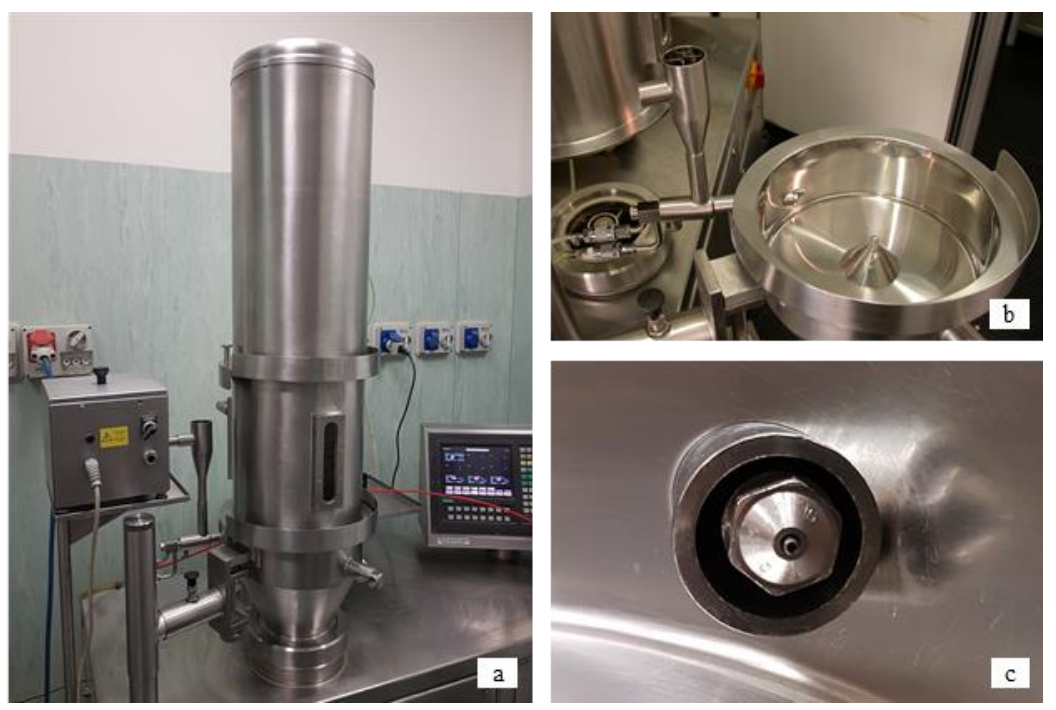

Figure 1. GPCG-1 fluid bed apparatus with rotor insert (a), open rotor insert equipped with powder feeder (b) and three-way nozzle with peripheral port for powder dosing (c).

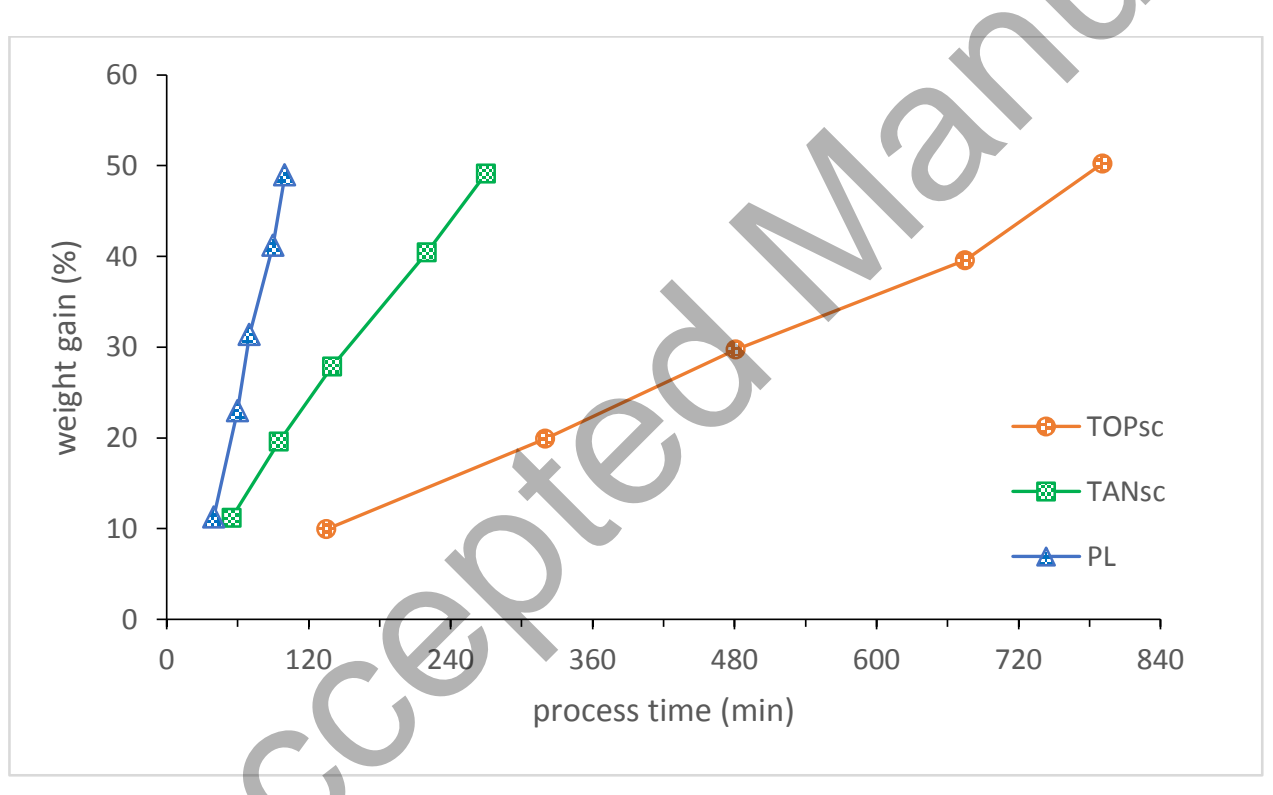

Figure 2. Relationship between coating level and process time with the different techniques under investigation. 

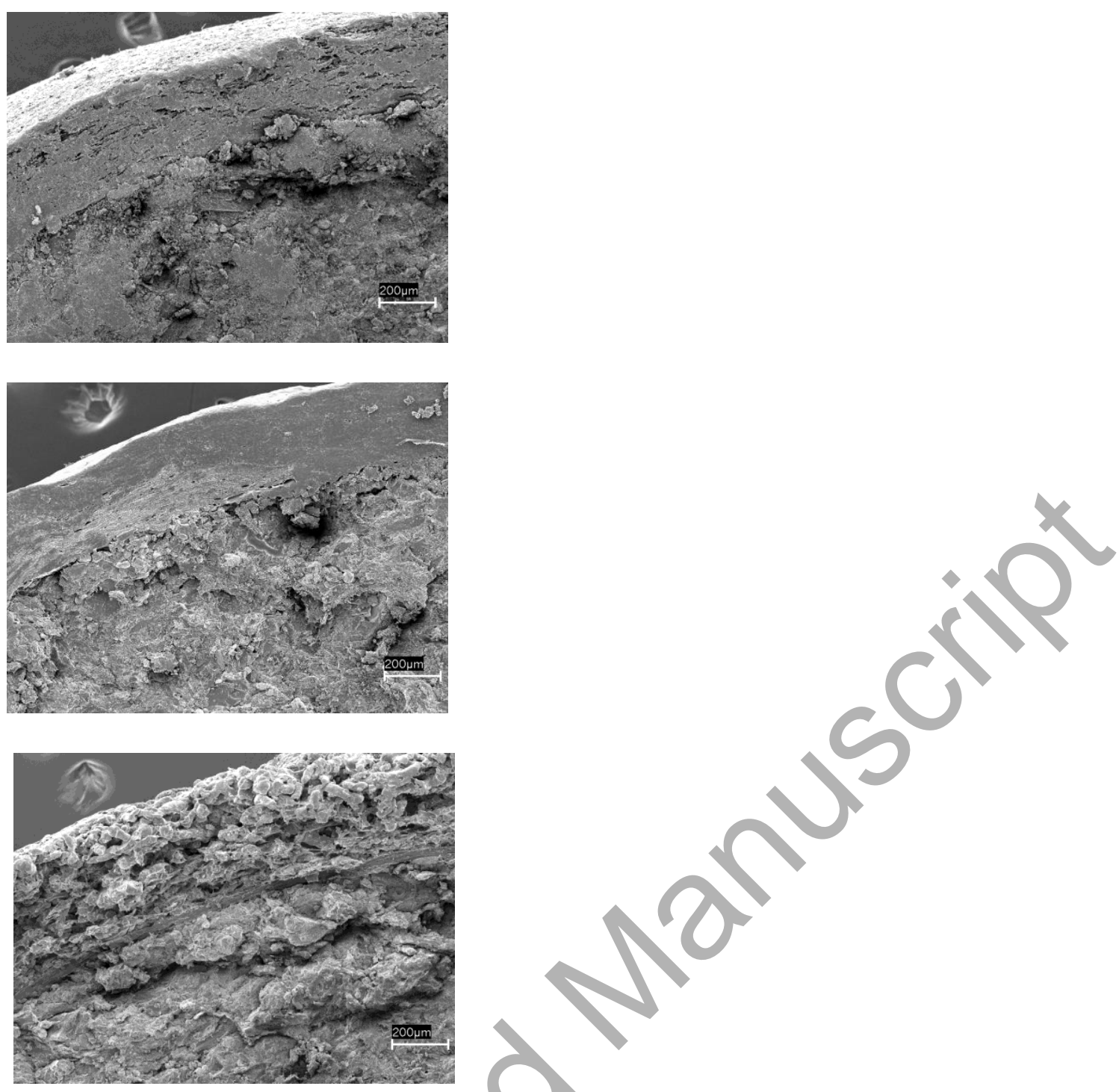

Figure 3. SEM photomicrographs of cross-sectioned coated units having $40 \%$ w.g. obtained by TOPsc, (top) TANsc (middle) and PL (bottom).

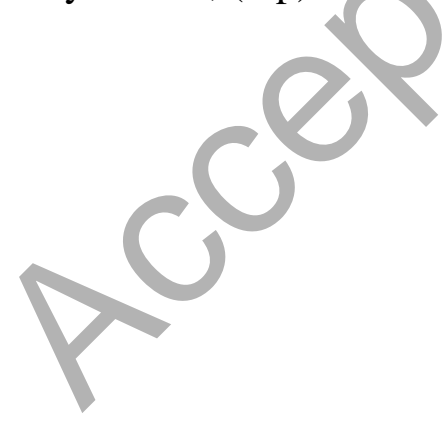




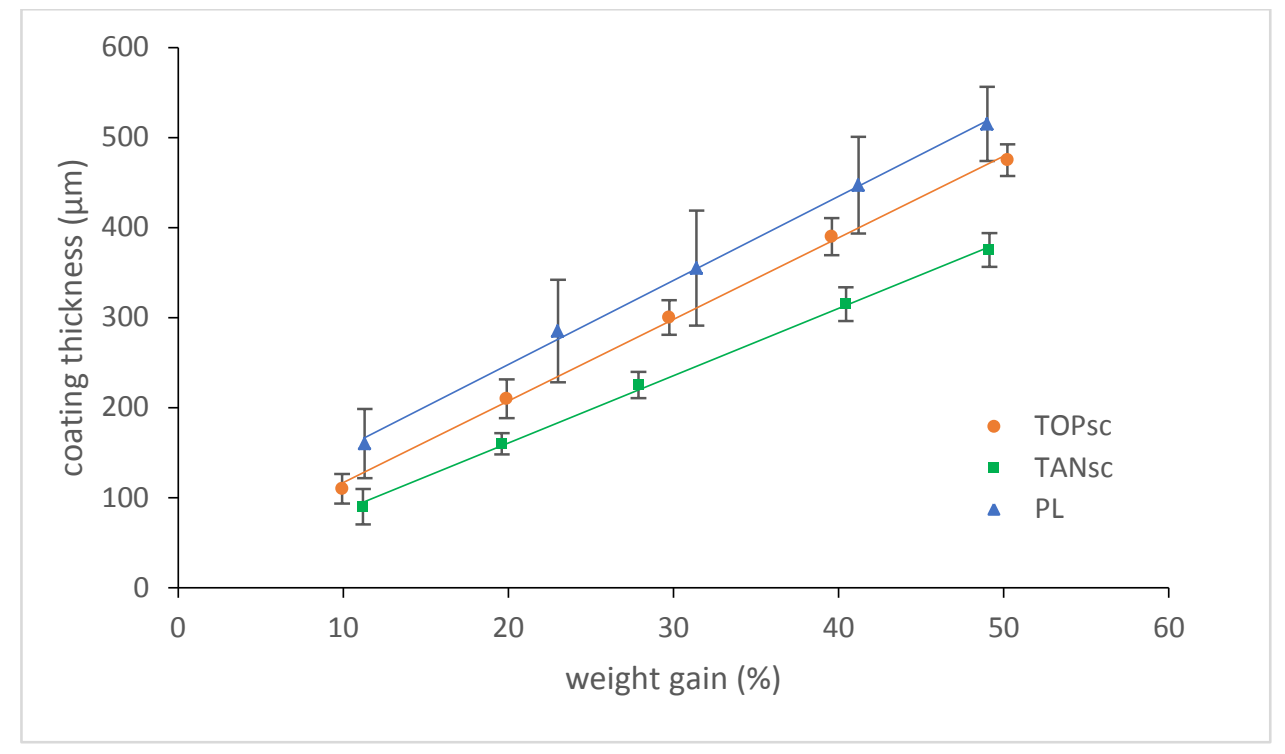

Figure 4. Relationship between coat thickness and weight gain of systems obtained by the different techniques under investigation. 


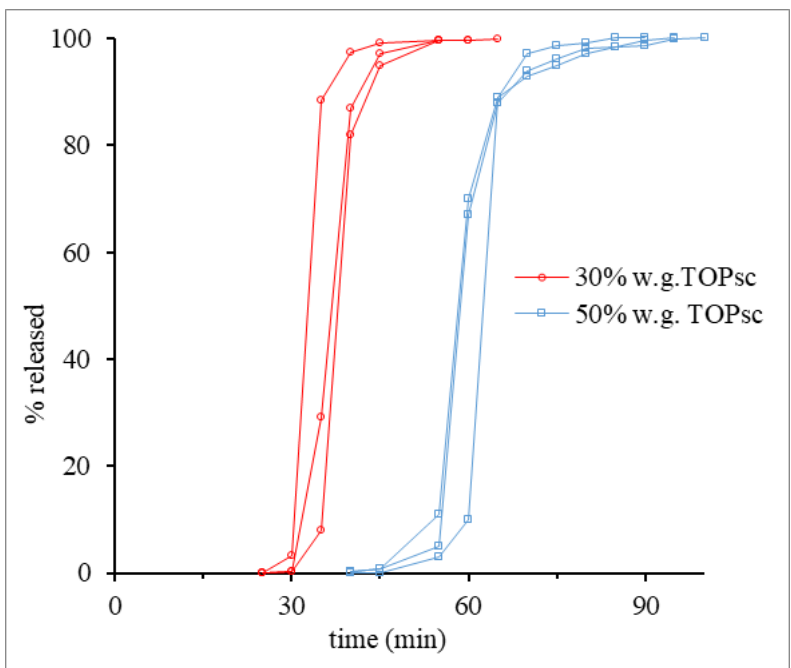

(a)

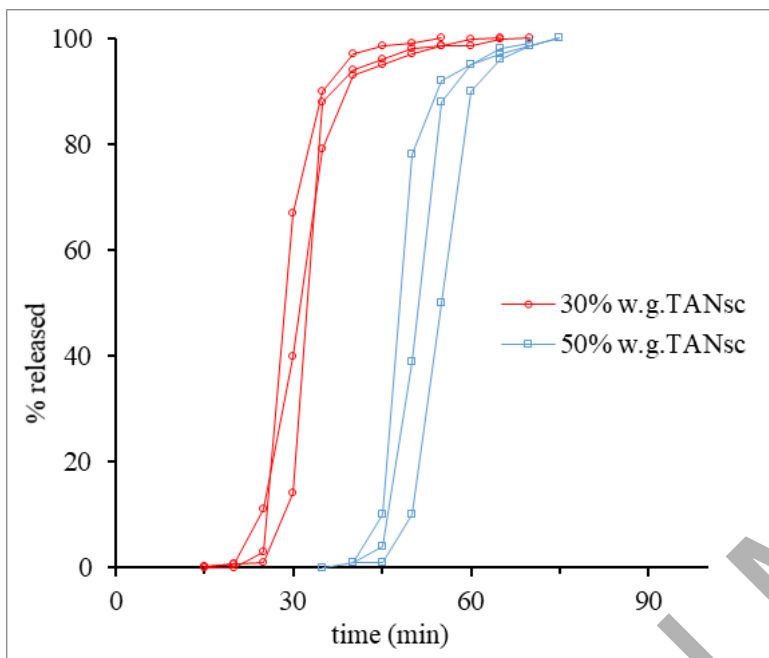

(b)

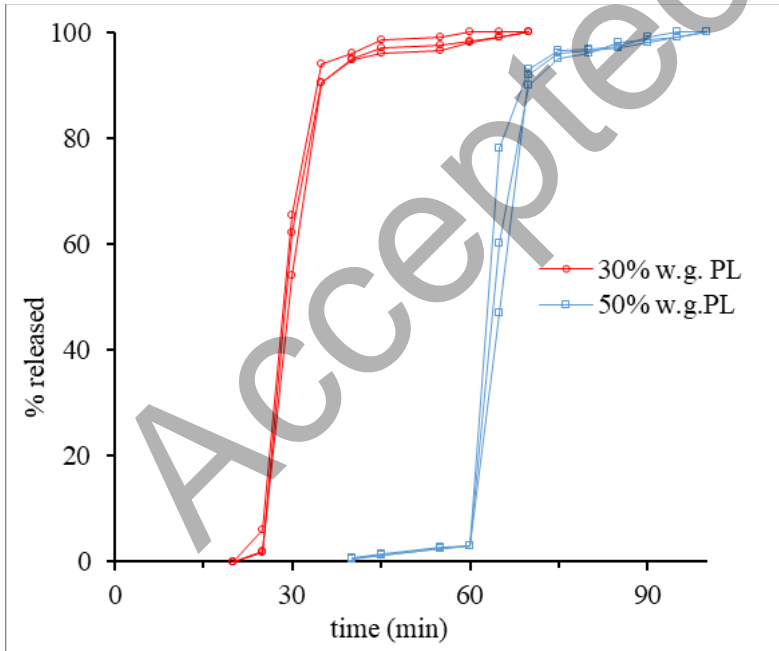

(c)

Figure 5 Release profiles of AMP from coated systems having different weight gain, obtained by (a) TOPsc, (b) TANsc and (c) PL. 


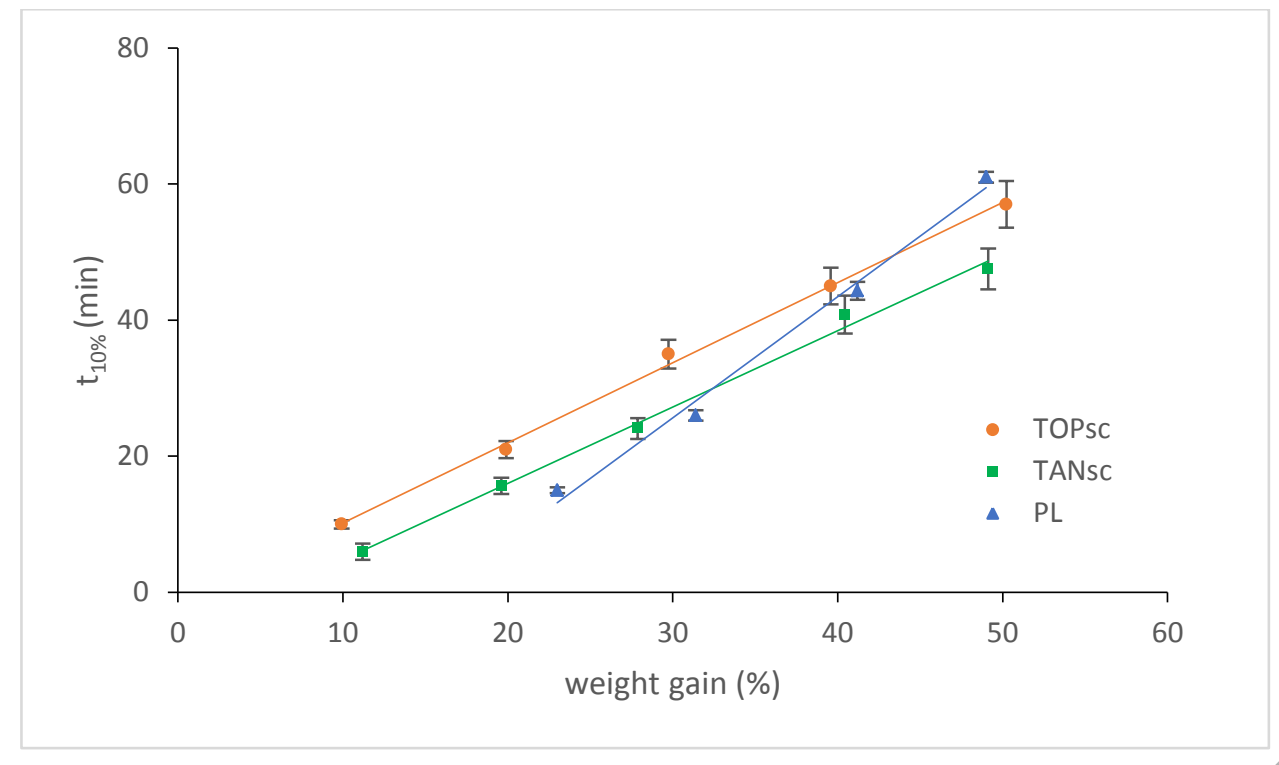

Figure 6: Relationship between lag time and weight gain of systems obtained by the different techniques under investigation. 
Table I. Set and derived process parameters relevant to the different coating techniques under investigation.

\begin{tabular}{|c|c|c|c|c|c|}
\hline & TOPsc & $\mathrm{TAN}_{\mathrm{SC}}$ & & PL & \\
\hline & & & sealing & $\begin{array}{l}\text { powder } \\
\text { layering }\end{array}$ & drying \\
\hline Inlet air temperature $\left({ }^{\circ} \mathrm{C}\right)$ & 60 & 60 & 60 & 30 & 65 \\
\hline Product temperature $\left({ }^{\circ} \mathrm{C}\right)$ & $45-50$ & $37-42$ & $40-45$ & $24-26$ & $27-40$ \\
\hline Outlet air temperature $\left({ }^{\circ} \mathrm{C}\right)$ & $40-50$ & $38-48$ & $36-45$ & $22-26$ & $25-38$ \\
\hline $\begin{array}{l}\text { Coating solution } \\
\text { temperature }\left({ }^{\circ} \mathrm{C}\right)\end{array}$ & 55 & 55 & & & \\
\hline Rotating disk speed (rpm) & & 500 & 500 & 5 & 500 \\
\hline $\begin{array}{l}\text { Nebulization air pressure } \\
\text { (bar) }\end{array}$ & 3.5 & 1.8 & 1.2 & & \\
\hline Airflow rate $\left(\mathrm{m}^{3} / \mathrm{h}\right)$ & 100 & 100 & & 5 & 100 \\
\hline Liquid spray rate (g/min) & 10.5 & 25.0 & & 12.0 & - \\
\hline Powder dosing rate $(\mathrm{g} / \mathrm{min})$ & & & & 6 & - \\
\hline $\begin{array}{l}\text { Amount of coating } \\
\text { solution }^{*}(g)\end{array}$ & 8120 & & & & \\
\hline $\begin{array}{l}\text { Amount of binding } \\
\text { solution }{ }^{*}(g)\end{array}$ & & & 360 & 850 & - \\
\hline $\begin{array}{l}\text { Amount of powder } \\
\text { formulation }(g)^{*}\end{array}$ & & - & - & 420 & - \\
\hline Process time $(\min )^{*}$ & & 270 & 30 & 70 & 30 \\
\hline Process yield (\%) & 72 & 85 & & 94 & \\
\hline
\end{tabular}

to $50 \% \mathrm{w} . \mathrm{g}$. 
Table II. Weight and coat thickness of systems having increasing coating levels obtained by the different techniques under investigation (standard deviations in brackets).

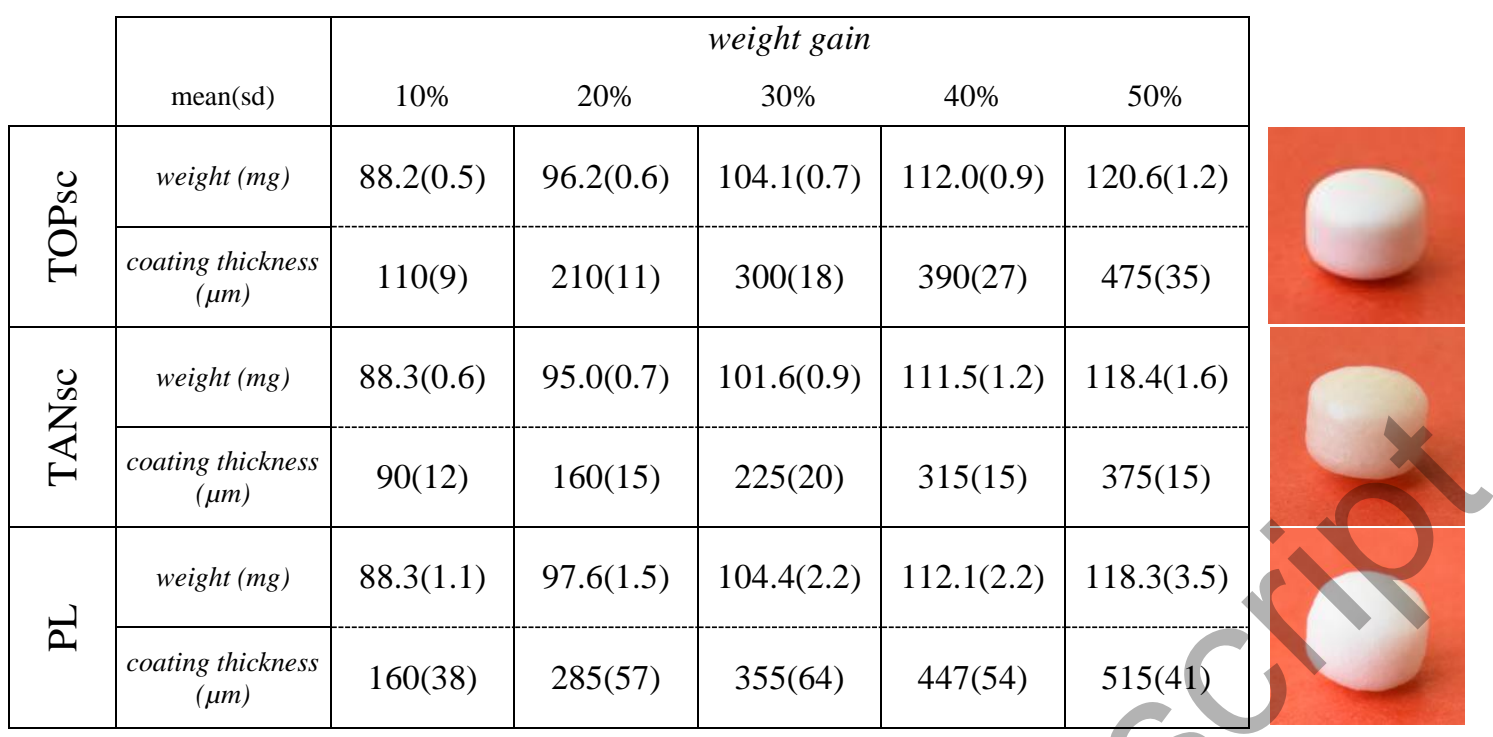

